\title{
Fotosammlung Robert Sennecke
}

Vermutlich ist es der schnellen Reaktion auf das Angebot zu verdanken, dass die Bibliothek für Zeitgeschichte (BfZ) im Sommer 2015 den Zuschlag erhielt. Zum Verkauf stand eine Sammlung von ca. 500 Positiven und 190 Negativen, dazu weitere biographische Materialien aus dem Nachlass des Pressefotografen Robert Sennecke. Dass der Erwerb gelang, ist für die BfZ sehr erfreulich, denn eine Sammlung dieser Qualität aus den Anfängen der Kriegs- und Pressefotografie kommt nur noch selten auf den Markt. Für das Sammelgebiet der BfZ sind die Dokumente in mehrfacher Hinsicht einschlägig und damit eine wertvolle Ergänzung ihres Bestands.

Ein Teil der aus Familienbesitz übernommenen Fotografien zeigt Szenen aus dem Berliner Familienleben vor und nach dem Ersten Weltkrieg bis in die späten 1920er Jahre (Feste, Ausflüge und Ferienreisen). Von Robert Senneckes Auftrags-Reisen der Vorkriegszeit sind Bilder und Briefe einer Mittelmeerreise an Bord des Luxusdampfers Oceana von 1908 erhalten: Ansichten von San Sebastian, Madeira und den Kanaren. Unter den Familienfotos finden sich prägnante Beispiele früher Porträtfotografie.

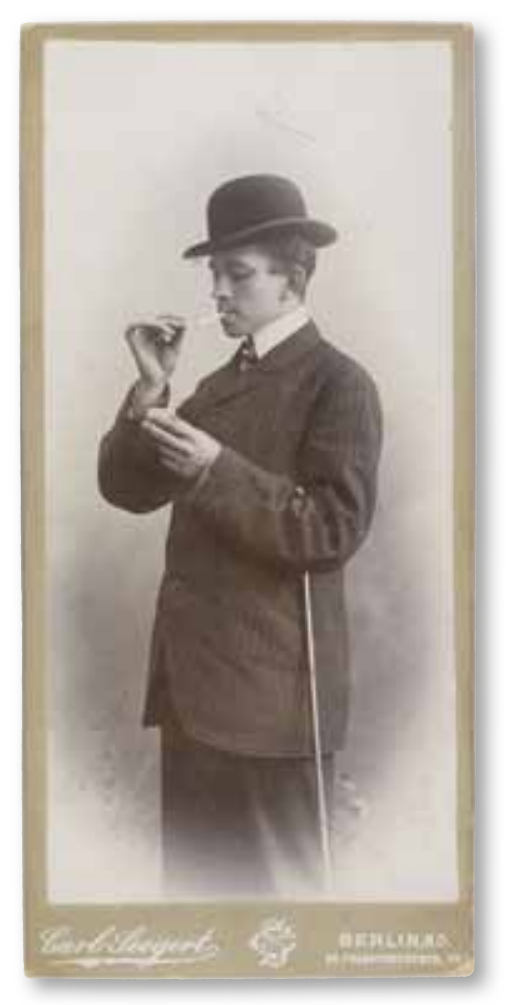

Abb. 1: Robert Sennecke, um 1905
Auf über 70

Aufnahmen ist

Robert Sennecke selbst zu sehen. Einige Bilder zeigen ihn während seiner Militärzeit, andere als Sportler. Sennecke, der 1906 als Marathonläufer an den Olympischen Spielen teilnahm, ist als Läufer, Radfahrer, Fußballer, Skifahrer, als Motoradrennfahrer und am Steuer eines Cabriolets zu sehen.
Von seinen eigenen Arbeiten als Sportfotograf sind nur wenige Bilder dabei. Sie befinden sich heute im Carl- und Liselott Diem-Archiv der Sporthochschule Köln.

Die zeitgeschichtlich interessantesten Aufnahmen der Neuerwerbung stammen von Robert Senneckes Reisen und Einsatzorten während des Ersten Weltkriegs. Eine weitere Besonderheit dieser Kriegsfotos ist die Aufnahmetechnik: 230 Bilder wurden mit einer stereoskopischen Kamera aufgenommen und vermitteln dadurch einen faszinierenden räumlichen Eindruck. Der Betrachter - man benötigt eine entsprechende Brille - fühlt sich durch diesen Effekt der Stereofotografie in die Aufnahme nahezu hineingezogen. Die sehr spezielle Bildsprache ermöglicht so einen ungewohnt intensiven Blick auf den Kriegsschauplatz.

Robert Sennecke, geboren 1885 in Pyritz/Pommern, stieg schon vor dem Ersten Weltkrieg in das Geschäft mit den aktuellen Bildern ein. Er beteiligte sich im Laufe seiner beruflichen Karriere an verschiedenen Bildagenturen und gründete einen eigenen Bildverlag.

Die erste deutsche Agentur für Pressefotos war 1900 in Berlin eröffnet worden. Um den Bedarf der illustrierten Blätter stillen zu können, entwickelte sich rasch der Beruf des Fotoreporters als ein Spezialgebiet der Fotografenzunft. Im Jahr 1910 existierten in Berlin bereits über zwanzig Firmen, die sich

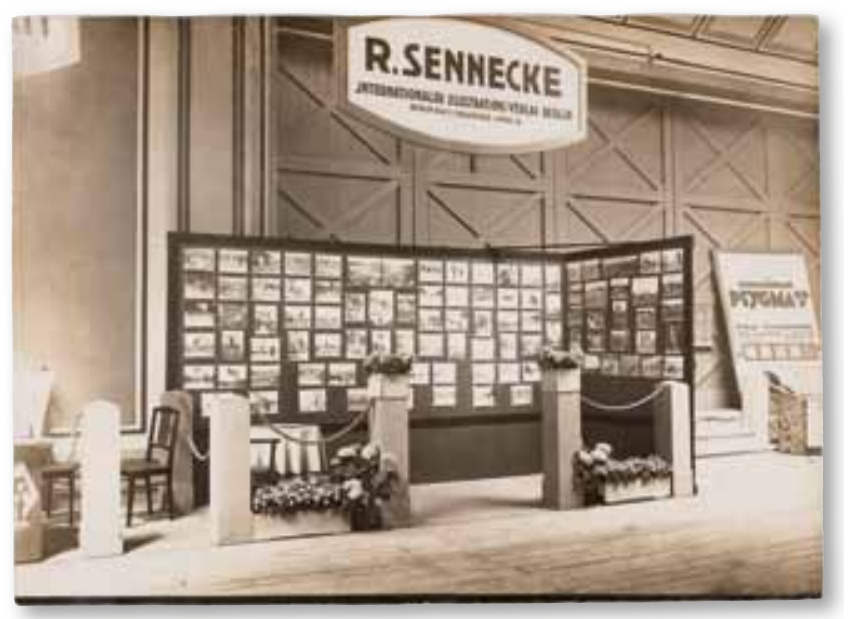

Abb. 2: Präsentation von Sportfotografien auf einem Messestand 
auf Pressefotografie ausgerichtet hatten. Eine der größten war die Firma „Robert Sennecke. Internationaler Illustrations-Verlag“. Mehrere Fotografen waren hier beschäftigt, außerdem wurden ausländische Bilder vertrieben und Pressefotos ins Ausland geliefert.

1915 musste Robert Sennecke, inzwischen verheiratet und Vater einer Tochter, in den Krieg ziehen. Es gelang ihm aber, in seinem erlernten Beruf Verwendung zu finden: als Kriegsfotograf. Auch seiner Firma in Berlin eröffnete sich mit Beginn des Ersten Weltkriegs ein neues Arbeitsfeld, sie konzentrierte sich nun auf den Vertrieb von Bildern der Kriegsschauplätze. In der Fotosammlung der BfZ befinden sich hunderte von Pressebildern, die den Stempel „Robert Sennecke. Internationaler Illustrations-Verlag" aufweisen und während des Ersten Weltkriegs von der Weltkriegsbücherei (später BfZ) bezogen wurden. Von welchen Fotografen sie aufgenommen worden sind, ob sie etwa von Sennecke selbst stammen, ist dort leider nicht festgehalten.

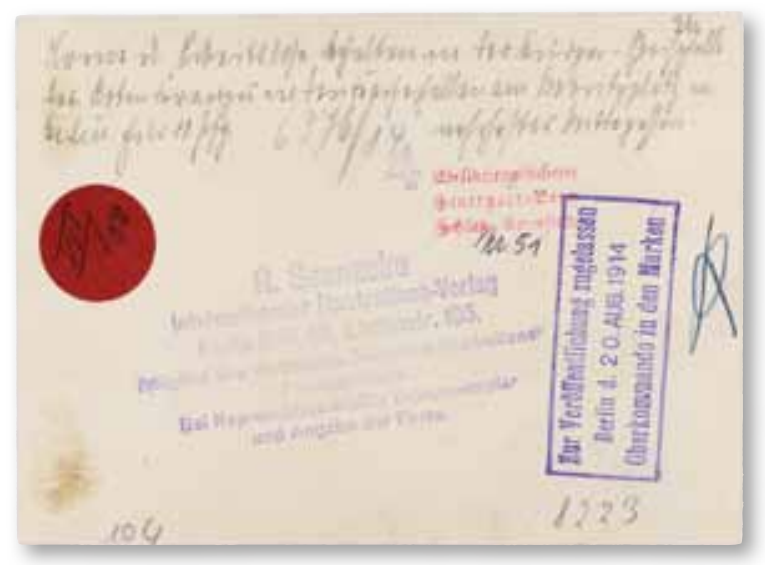

Abb. 3: Stempel der Bildagentur Sennecke auf einem Pressefoto von 1914

Den Materialien aus dem Nachlass folgend - eine Chronologie seines Kriegseinsatzes ist nicht bekannt - fotografierte Sennecke in Frankreich und in Flandern. Von der Vielzahl der Kriegspressebilder von

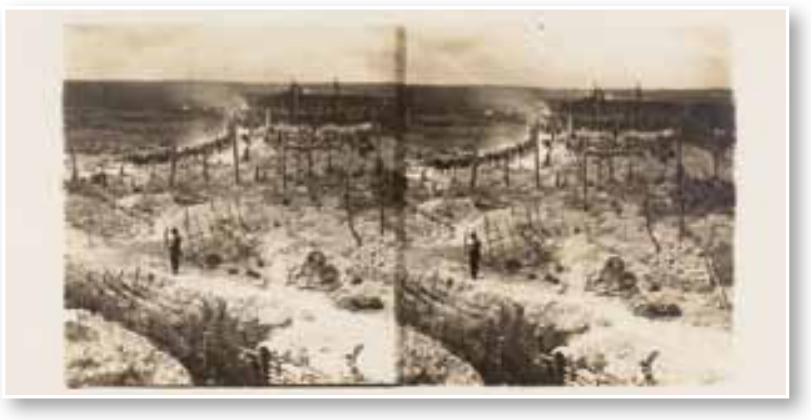

Abb. 4: Schlachtfeld der Westfront (Stereofotografie) der Westfront unterscheidet diese Aufnahmen nicht die Motivwahl, sondern der Blick des Fotografen. Sennecke zeigt auf fast allen Bildern Personen. Der Soldat wird als Element einer zerstörten Landschaft, inmitten von Chaos dargestellt.

Auf bemerkenswerte Weise gelingt es ihm in manchen Fällen, eine Bewegung einzufangen und dem Foto dadurch Dynamik zu verleihen - für die kameratechnischen Möglichkeiten der Zeit sicher eine Herausforderung.

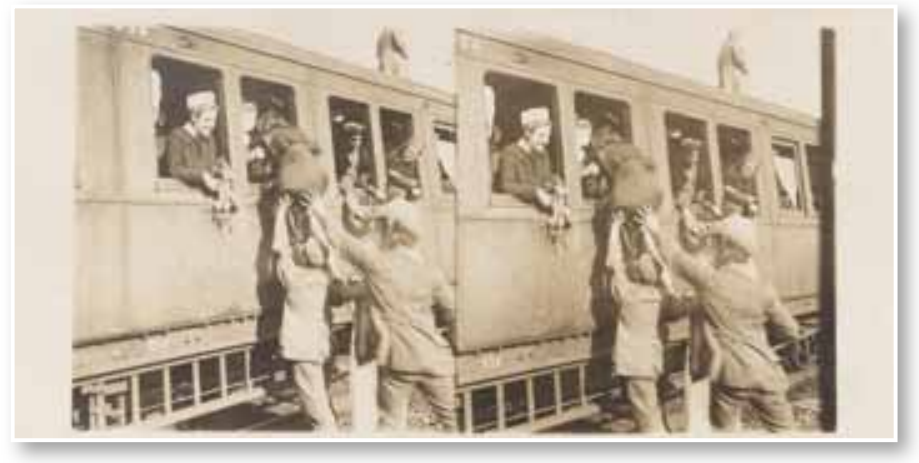

Abb. 5: Krankenschwestern besteigen den Zug nach Brüssel (Stereofotografie)

1916/1917 war Sennecke in Griechenland und der Türkei unterwegs. Auf seinen Reisestationen Athen und Konstantinopel erwies er sich als aufmerksamer Beobachter auch des Alltäglichen. Im Jahr 1917 wurde er als Fotograf im Rang eines Gardefüsiliers dem „Filmtrupp Orient" der "Deutschen Militärmission Constantinopel" zugeteilt und bereiste Palästina - ein Ausweis mit Passbild hat sich unter den Dokumenten erhalten. Seine Bildmotive im Osmanischen Reich sind Militärparaden und Straßenszenen in Konstantinopel, aber auch das Auftreten der deutschen Soldaten in ländlicher Umgebung bei der Begegnung mit einheimischen Zivilisten. Senneckes Arbeit wurde gewürdigt, er erhielt die osmanische Kriegsmedaille „Eiserner Halbmond".

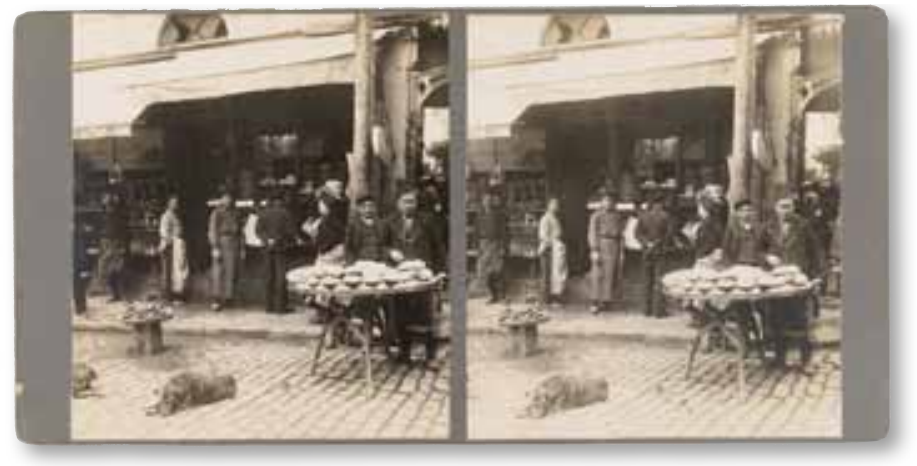

Abb. 6: Konstantinopel, 1916/17 (Stereofotografie) 


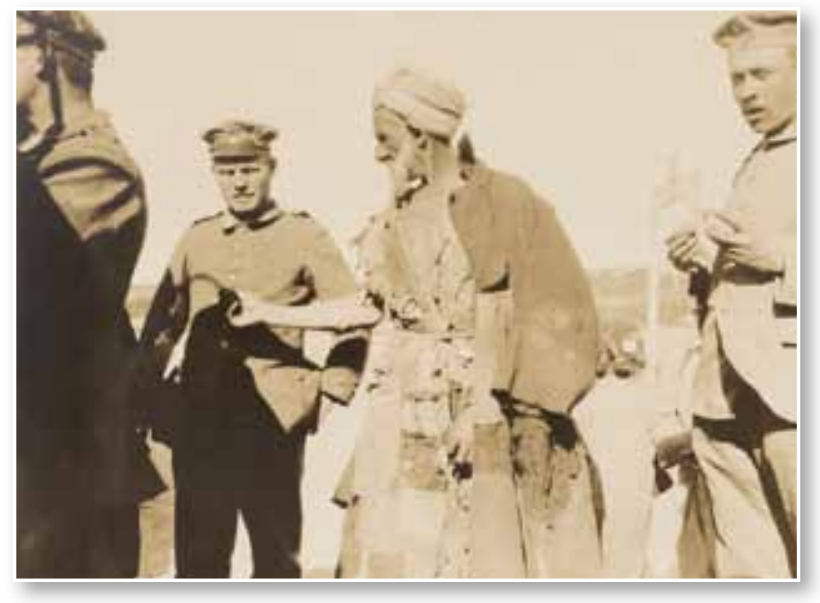

Abb. 7: Deutsche Soldaten in der Türkei, 1916/17
Am 30. November 1918 entließ der Soldatenrat des Preußischen Bild- und Filmamts den Füsilier Sennecke mit "1 Tuchrock, 1 Tuchhose, 1 Mantel, 1 Paar Schnürschuh" und 50 Mark Entlassungsgeld aus dem Kriegsdienst. Auch seine Berliner Firma hatte Robert Sennecke über den Krieg retten können. Viele der heute bekannten Aufnahmen der Novemberrevolution wurden von Senneckes Bildagentur am Halleschen Ufer 9 vertrieben. Persönlich reiste Sennecke nach München, um das dortige Revolutionsgeschehen gleichfalls mit der Kamera festzuhalten. Aus dieser Epoche sind im neu erworbenen Konvolut aber leider keine Presse-Fotografien Senneckes enthalten.

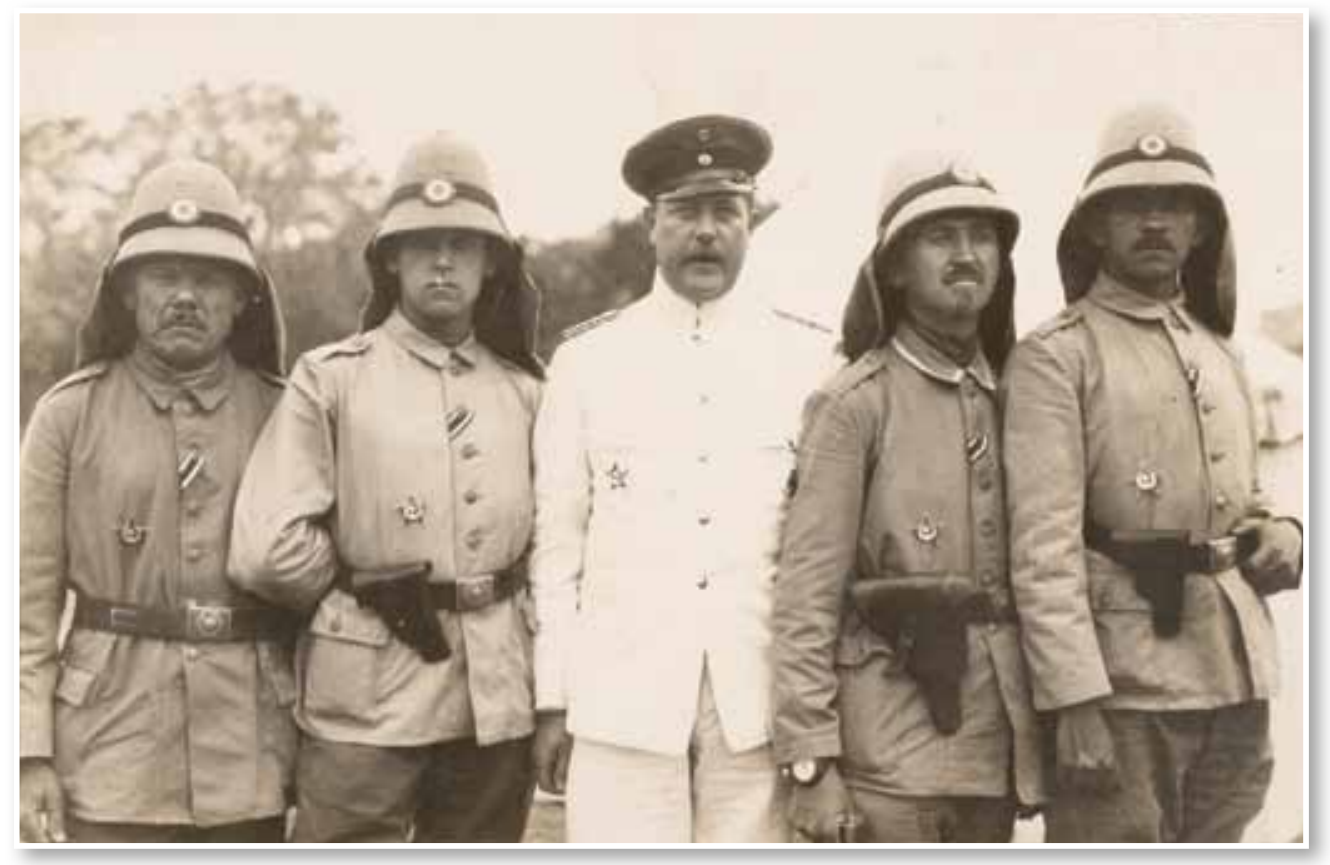

Noch während der

Weimarer Republik gehörte Senneckes "Internationaler Illustrations-Verlag" zu den großen Bildlieferanten, die täglich an hunderte Zeitungsredaktionen im In- und Ausland Fotografien versandten. 1928 erkrankte Robert Sennecke aufgrund einer Malariainfektion, die er sich im Krieg zugezogen hatte, und musste daraufhin die
Firmenleitung aufgeben. Seine Tochter scheiterte bald mit der Weiterführung des Verlags und konnte schließlich den Konkurs nicht abwehren. Robert Sennecke starb 1940 in der Landesanstalt Lübben. Die Familie geht davon aus, dass er zum Euthanasieopfer wurde.

Irina Renz 